\title{
hsa_circ_0000231 Promotes colorectal cancer cell growth through upregulation of CCND2 by IGF2BP3/miR-375 dual pathway
}

Wei Zhang ${ }^{1,2,3+}$, Bo Wang ${ }^{1,3+}$, Yilin Lin ${ }^{1,2,3+}$, Yang Yang ${ }^{1,3}$, Zhen Zhang ${ }^{2,3}$, Quan Wang ${ }^{2,3}$, Haoran Zhang ${ }^{2,3}$, Kewei Jiang ${ }^{1}$, Yingjiang Ye ${ }^{1}$, Shan Wang ${ }^{2,3}$ and Zhanlong Shen ${ }^{1,2,3^{*}}$

\begin{abstract}
Background: Circular RNAs (circRNAs) have emerged as vital regulators of the initiation and progression of diverse kinds of human cancers. In this study, we explored the role of hsa_circ_0000231 and its downstream pathway in CRC.

Methods: The expression profile of circRNAs in 5 pairs of CRC tissues and adjacent normal tissues were analyzed by Microarray. Quantitative real-time PCR and in situ hybridization and Base Scope Assay were used to determine the level and prognostic values of hsa_circ_0000231. Then, functional experiments in vitro and in vivo were performed to investigate the effects of hsa_circ_0000231 on cell proliferation. Mechanistically, fluorescent in situ hybridization, dual luciferase reporter assay, RNA pull-down and RNA immunoprecipitation experiments were performed to confirm the interaction between hsa_circ_0000231 and /GF2BP3 or has_miR-375.
\end{abstract}

Results: We acquired data through circRNA microarray profiles, showing that the expression of hsa_circ_0000231 was upregulated in CRC primary tissues compared to adjacent normal tissues, which was indicated poor prognosis of patients with CRC. Functional analysis indicated that inhibition of hsa_circ_0000231 in CRC cell lines could suppress CRC cell proliferation as well as tumorigenesis in vitro and in vivo. The mechanistic analysis showed that $h s a_{-}$ circ_0000231 might, on the one hand, act as a competing endogenous RNA of miR-375 to promote cyclin D2 (CCND2) and, on the other hand, bind to the IGF2BP3 protein to prevent CCND2 degradation.

Conclusions: The findings suggested that hsa_circ_0000231 facilitated CRC progression by sponging miR-375 or binding to IGF2BP3 to modulate CCND2, implying that hsa_circ_0000231 might be a potential new diagnostic and therapeutic biomarker of CRC.

Keywords: Colorectal cancer, CircRNA, IGF2BP3, miR-375, CCND2

\section{Background}

Colorectal cancer (CRC) is the third most commonly diagnosed cancer in men and the fourth most commonly diagnosed cancer in women worldwide [1]. The annual new cases of CRC account for its third place among

\footnotetext{
*Correspondence: shenzhanlong@pkuph.edu.cn

tWei Zhang, Bo Wang and Yilin Lin contributed equally to this work

1 Department of Gastroenterological Surgery, Peking University People's Hospital, Beijing 100044, People's Republic of China

Full list of author information is available at the end of the article
}

malignant tumors, and the related deaths account for the fourth place [2]. CRC was the fifth most commonly diagnosed cancer and the fifth most common cause of death by cancer in China in 2014, with an age-standardized incidence rate of 17.52 per 100,000 and age-standardized mortality rate of 7.91 per 100,000 [3].

$\mathrm{CRC}$ has become a major disease seriously threatening human health and posing a huge social and economic burden [4]. The treatment of CRC mainly adopts a comprehensive treatment mode centered on surgery,

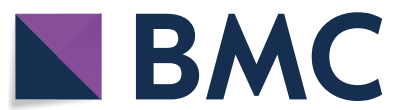

(c) The Author(s) 2022. Open Access This article is licensed under a Creative Commons Attribution 4.0 International License, which permits use, sharing, adaptation, distribution and reproduction in any medium or format, as long as you give appropriate credit to the original author(s) and the source, provide a link to the Creative Commons licence, and indicate if changes were made. The images or other third party material in this article are included in the article's Creative Commons licence, unless indicated otherwise in a credit line to the material. If material is not included in the article's Creative Commons licence and your intended use is not permitted by statutory regulation or exceeds the permitted use, you will need to obtain permission directly from the copyright holder. To view a copy of this licence, visit http://creativecommons.org/licenses/by/4.0/. The Creative Commons Public Domain Dedication waiver (http://creativecommons.org/publicdomain/zero/1.0/) applies to the data made available in this article, unless otherwise stated in a credit line to the data. 
but the curative effect is still unsatisfactory. The molecular mechanism underlying the development of CRC is not completely clear, which is one of the important reasons for its high incidence and poor prognosis [5]. Recent studies have shown the importance of a new family of noncoding RNAs, circular RNAs (circRNAs), in the molecular regulation of tumorigenesis and progression. They have a covalently closed circular structure at the $3^{\prime}$-untranslated region (UTR) and $5^{\prime}$-UTR, are widely and diversely present in eukaryotic cells, and have an endogenous RNA molecule that regulates gene expression [6-8]. The circRNA functions mainly via the endogenous RNA (ceRNA) mechanism, that is, circRNA acts as a "molecular sponge" and competitively binds to microRNA (miRNA) through a miRNA response element (MRE) to inhibit miRNA function. MiRNAs degrade or inhibit the expression of target genes through the RNAinduced silencing complex (RISC), which relies on the Argonaute2 (AGO2) protein [9].

MicroRNAs (miRNAs) are small noncoding RNAs with a size of 18-25 nucleotides, which function as post-transcriptional regulators of target mRNAs [10]. The increase in the expression of oncogenic miRNAs in cancer leads to the downregulation of tumor-suppressive genes. In contrast, the decrease in the expression of tumor-suppressive miRNAs enhances the expression of oncogenes. The findings indicated that miRNAs participated in the tumorigenesis and progression of various cancers, including colorectal cancer [11-13]. Also, circRNAs could bind to RNA-protective proteins [14]. However, the biological functions of most circRNAs in the pathogenesis and progression of $\mathrm{CRC}$ and the underlying mechanisms remain largely unclear.

The present study found 425 significantly differentially expressed circRNAs in CRC tissues. The $h s a_{-}$ circ_0000231 upregulation was further discovered to sponge $m i R-375$ and IGF2BP3 and hence regulate CRC progression. The hsa_circ_0000231 is vital in the development of CRC and participates in the molecular regulation of CRC by regulating the cell cycle.

\section{Methods}

\section{CircRNA microarray}

Five pairs of CRC tumor tissues and corresponding adjacent noncancerous tissues were used for circRNA microarrays. The specimens were obtained from patients undergoing surgery in the Peking University People's Hospital in 2014; detailed information is shown in Table 1.

\section{Patients and samples}

160 CRC patients who were diagnosed and underwent surgery in Peking University People's Hospital between
Table 1 Clinicopathological features for circRNA microarray

\begin{tabular}{lll}
\hline & Numbers & Percent (\%) \\
\hline Gender & & \\
Male & 4 & 80 \\
$\quad$ Female & 1 & 20 \\
Age at diagnosis & \\
$\quad \leq 60$ & 2 & 40 \\
$>60$ & 3 & 60 \\
Tumor size (cm) & & \\
$\leq 5$ & 5 & 100 \\
$>5$ & 0 & 0 \\
Differentiation & & 0 \\
Well-moderate & 0 & 0 \\
Poor & 0 & \\
Depth of invasion & & 0 \\
T1-T2 & 0 & 0 \\
T3-T4 & 0 & 40 \\
Lymph node metastasis & & 60 \\
No & 2 & 40 \\
Yes & 3 & 60 \\
TNM stage & 2 & \\
I-II & 3 & \\
III-IV & & \\
\hline
\end{tabular}

2014 and 2017 were included in this study. Fresh colorectal tumor tissues and matched normal colorectal mucosa tissues were obtained from all the 160 patients. The specimens were obtained and immediately frozen in liquid nitrogen and stored at $-80^{\circ} \mathrm{C}$ until RNA or protein extraction. This study was performed according to the recommendations in the Guide for the Chinese Ethics Review Committees. The protocol was approved by the Ethics Committee of Peking University People's Hospital. Written informed consent was obtained from each subject. The animal experiment was carried out under ethics approval of Peking University People's Hospital.

\section{Cell culture}

Human colorectal cancer SW480, SW620, HCT116, HT29, RKO, LS174T, COLO-215, HCT8 and LoVo cell lines were purchased from American Type Culture Collection (Manassas, USA). Human normal intestinal epithelial cells NCM460 were purchased from INCELL Corporation LLC (San Antonio, USA). HCT116, HT29, RKO, LoVo, COLO-215, HCT8 and NCM460 cells are cultured in RPMI-1640. LS174T cells were cultured in DMEM. SW480 and SW620 were cultured in Leibovitz's $\mathrm{L}-15$ medium. All cells were cultured at $37^{\circ} \mathrm{C}$ containing $5 \% \mathrm{CO}_{2}$. All medium were supplemented with $10 \%$ fetal bovine serum (FBS, Gibco, USA). 


\section{Cellular fluorescence in situ hybridization}

The cells were fixed with $4 \%$ paraformaldehyde for $15 \mathrm{~min}$ and washed with phosphate-buffered saline (PBS) three times for 5 min each time. Further, they were permeabilized with $0.5 \%$ Triton $\mathrm{X}-100$ at room temperature for $20 \mathrm{~min}$ and washed with PBS three times for 3 min each time. Then, pepsin freshly diluted with 3\% citric acid was added and digested at room temperature for $15 \mathrm{~min}$. Subsequently, the nucleic acid fragment was exposed, rinsed with PBS, mixed with 20 $\mu \mathrm{L}$ of a pre-hybrid solution, and pre-hybridized at $50{ }^{\circ} \mathrm{C}$ for 2-4 h. The hsa_circ_0000231 or miR375-specific probe hybridized at a constant temperature of $50{ }^{\circ} \mathrm{C}$. Hybridization was carried out using SSC at $37^{\circ} \mathrm{C}$, biotinylated mouse anti-digoxigenin was added dropwise, the fragment was washed with PBS three times for 3 min each time, and the excess solution was absorbed with the absorbent paper. After adding DAPI stain for $10 \mathrm{~min}$, the specimen was subjected to nuclear staining and washed three times with PBS for 3 min each time; the excess solution was absorbed by the absorbent paper. The specimen was sealed with a liquid containing a fluorescent quencher, and the image was observed under a fluorescence microscope.

\section{BaseScope assay}

BaseScope assay was performed following the manufacturer's protocols (Advanced Cell Diagnostics, CA, USA). The tissues were sectioned at $5-\mu \mathrm{m}$ thickness, placed onto Superfrost Plus slides (Fisher Scientific, Loughborough, UK), and allowed to dry overnight at $25{ }^{\circ} \mathrm{C}$. The sections were then baked at $60{ }^{\circ} \mathrm{C}$ for $1 \mathrm{~h}$ before deparaffinized in xylene (twice for $5 \mathrm{~min}$ ) and ethanol (twice for $2 \mathrm{~min}$ ), and then dried by baking at $60{ }^{\circ} \mathrm{C}$ for $2 \mathrm{~min}$. Subsequently, hydrogen peroxide was applied for $10 \mathrm{~min}$ at $25{ }^{\circ} \mathrm{C}$, target retrieval was performed for $15 \mathrm{~min}$ at $100{ }^{\circ} \mathrm{C}$, and RNAscope Protease III was applied at $40{ }^{\circ} \mathrm{C}$ for $30 \mathrm{~min}$. The samples were rinsed twice in distilled water between treatments. BaseScope probes (Mm1700024F13Rik, cat\#709881) with positive controls (Hs-PPIB, cat \# 701031; DapB, cat \# 701011) were then applied, and the samples were incubated for $2 \mathrm{~h}$ at $40{ }^{\circ} \mathrm{C}$ in a HybEZ oven and then with reagents AMPO (30 min at $\left.40{ }^{\circ} \mathrm{C}\right)$, AMP1 $\left(15 \mathrm{~min}\right.$ at $\left.40{ }^{\circ} \mathrm{C}\right), \mathrm{AMP} 2(30 \mathrm{~min}$ at $\left.40{ }^{\circ} \mathrm{C}\right)$, AMP3 $\left(30 \mathrm{~min}\right.$ at $\left.40^{\circ} \mathrm{C}\right)$, AMP4 $\left(15 \mathrm{~min}\right.$ at $\left.40^{\circ} \mathrm{C}\right)$, AMP5 $\left(30 \mathrm{~min}\right.$ at $\left.25^{\circ} \mathrm{C}\right)$, and AMP6 $\left(15 \mathrm{~min}\right.$ at $\left.25^{\circ} \mathrm{C}\right)$. The slides were rinsed with wash buffer (twice for $2 \mathrm{~min}$ ) between AMP incubation steps. Finally, they were treated with Fast Red for $10 \mathrm{~min}$ at $25^{\circ} \mathrm{C}$ in the dark, counterstained with Gill's hematoxylin, dried for $15 \mathrm{~min}$ at $60{ }^{\circ} \mathrm{C}$, and mounted in Catamount permanent mounting medium (Vector Labs, CA, USA).

\section{RNA extraction and quantitative real-time polymerase} chain reaction

Total RNA from cell lines and tissue samples was extracted using TRIzol (Invitrogen, USA) following the manufacturer's instructions. For the plasma, the total RNAs were extracted using an mirVana PARISTM microRNA extraction kit (ABI, USA) following the manufacturer's protocols. For lncRNA quantification, GAPDH was used as internal control, and PrimeScript RT Master Mix (QIAGEN, Germany) was used for reverse transcription and real-time polymerase chain reaction (PCR). For circRNA expression detection, RNase $\mathrm{R}$ was added to remove linear RNA after the sample RNA was extracted, and then random primers were reverse transcribed into cDNA for further amplification. For the design of circRNA amplification primers, this study adopts the design method of trans circularization site primers. The primer sequences of all genes are shown in Additional file 1: Table S1. All reactions were performed in triplicate. The fold change for each gene relative to the control group was calculated using the $2^{-\Delta \Delta C t}$ method.

\section{Lentiviral short hairpin RNA particles}

Recombinant lentiviral particles expressing hsa circ_0000231 and normal control (NC) were obtained from GenePharm Co., Ltd. (Shanghai, China). SW480 cells were grown to approximately $40 \%$ confluence and infected with lentiviral particles in complete medium for $48 \mathrm{~h}$. They were co-treated with the cationic polymer polybrene $(8 \mu \mathrm{g} / \mathrm{ml}$ in water $)$ to increase the infection efficiency. Neither shRNA nor polybrene affected cell viability. Further, shRNA had no off-target effects, and did not affect cell adherence, shape, or viability at the indicated multiplicity of infection.

\section{Cell transfection}

For in vitro studies, siRNA interference sequences targeting hsa_circ_0000231 and the best transcript of $A R H$ GAP12 which hsa_circ_0000231 located at-mRNA (NM_018287) normal control (si-NC) were designed and synthesized (Ribobio, Guangzhou, China) to interfere with the expression of hsa_circ_0000231 or mRNA, which named si-circ_0000231 or si-mRNA, and a final concentration of $50 \mathrm{nM}$ was used for transient transfection. Similarly, mimics-NC and miR-375 mimics were designed and synthesized (Ribobio, Guangzhou, China) to overexpress $m i R$-375. Lipofectamine 3000 (Invitrogen, CA, USA) was used for transfection following the manufacturer's protocols.

For in vivo assays, the $h s a_{-}$circ_0000231 overexpression cell line was used. The hsa_circ_0000231 gene was cloned into a lentivirus vector LV-GFP-Puro, and SW480 cells 
were used for infection. Stable transfection cells were established by puromycin antibiotic selection for 7 days, with a concentration of $2.5 \mu \mathrm{g} / \mathrm{ml}$. The hsa_circ_0000231overexpressing cells and control cells were named LVhsa_circ_0000231(LV-circ) and LV-NC, respectively.

Transfection and grouping of cells: Cell transfection were performed in strict accordance with the instructions of Invitrogen's Lipofectamine 3000 Transfection Reagent when the cytoplasm was inoculated with a confluence of about $80 \%$. The Lipofectamine 3000 reagent was diluted with OPTI-MEM culture medium and mixed. The DNA expression plasmid to be transfected was diluted with OPTI-MEM culture medium and mixed with P3000 reagent. The diluted DNA expression plasmid was added in equal volume to each dilution of Lipofectamine 3000 reagent and incubated at room temperature for $5 \mathrm{~min}$. The DNA-liposome mixture was added to the cell suspension and carefully mixed. The culture was continued in a $5 \%$ $\mathrm{CO}_{2}$ incubator at $37^{\circ} \mathrm{C}$.

\section{Cell proliferation assay}

SW480 and SW620 cells $\left(3 \times 10^{3}\right.$ cells $)$ were seeded in complete medium in 96-well plates and infected with hsa_circ_0000231 siRNA. The cell proliferation assay was performed with a Cell Counting Kit 8 (CCK8) following the manufacturer's protocol, and cell proliferation was detected after $0,24,48,72$, and 96 h. The cells in each group were tested for five replicates. The cell proliferation was evaluated by the CCK- 8 method using a microplate reader (Molecular Devices, CA, USA) following the manufacturer's protocols to measure the absorbance.

For the colony formation assay, the transfected cells were seeded into each well of a 6-well plate on day 0 and then incubated for another 14 days. Then, the wells were fixed with $4 \%$ paraformaldehyde and stained with $0.1 \%$ crystal violet. The colonies so formed were counted and analyzed using Image J software.

\section{Western blot analysis}

RIPA lysis buffer (Solarbio, Beijing, China) to extract total protein. The proteins were quantified by the bicinchoninic acid (BCA) assay kit (Solarbio, Beijing, China). Add SDS loading buffer to the extracted total protein, and then boil it in $100^{\circ}$ water for five minutes for subsequent experiments. The obtained protein was electrophoresed in $12 \%$ sodium dodecyl sulfate-polyacrylamide gel electrophoresis (SDS-PAGE). PVDF membrane was used for electroporation. The protein electrophoresis was performed at a stable voltage of $100 \mathrm{~V}$, and the electroporation was performed at a stable current of $300 \mathrm{~mA}$ for $90 \mathrm{~min}$. After electroporation, soak the PVDF membrane in $5 \%$ skimmed milk and place it on a shaker for half an hour. Then add $5 \mathrm{ml}$ of CCND2 (1:1000), IGF2BP3
(1:1000) (Abcam, CA, USA), RB (1:500), and GAPDH (1:5000) (Cell Signaling Technology, MA, USA) rabbitderived primary antibody to the PVDF membrane and incubate overnight at $4^{\circ}$. After incubating overnight, collect the primary antibody, add Tris-Buffered Sal ine Tween 20 (TBST) and wash three times for 15 min each time. Add goat anti-rabbit (Solarbio, Beijing, China, 1:5000) and incubate for $1 \mathrm{~h}$, then continue to wash with TBST three times. Finally, add electrochemiluminescence (Solarbio, Beijing, China) liquid to expose in the exposure instrument. The antibodies used in the experiments are shown in Additional file 2: Table S2.

\section{Luciferase reporter assay}

The hsa_circ_0000231 and CCND2 fragments containing two putative wild-type or mutated miR-375-binding sites were amplified by PCR and cloned downstream of the luciferase gene in the pGL3 vector (Promega, WI, USA). The constructed reporter vectors were verified by sequencing. Luciferase reporter assays were performed by transiently co-transfecting SW480 cells in 24-well plates with the reporter vectors, $m i R-375$, and the Renilla luciferase construct using Lipofectamine 2000 (Invitrogen, MA, USA). After 48-h transfection, the cells were harvested, and luciferase activity was measured using a dual-luciferase reporter assay system (Promega) and normalized to that of Renilla luciferase.

\section{Biotin-labeled RNA pull-down and mass spectrometry analysis}

Biotin-labeled RNA for the linear sequence of hsa circ_0000231 and CCND2 was generated by an in vitro transcription reaction with the Biotin RNA Labeling Mix (Roche, Mannheim, Germany) and T7 RNA polymerase (Roche), and then treated with RNase-free DNase I (TaKaRa, Japan). After incubation with the oligonucleotide targeting circular junction, the liner probe was circularized using T4 RNA ligase I and treated with RNase R. After purification with a RNeasy Mini Kit (Qiagen, Inc., CA, USA), the biotin-labeled RNA probe $(3 \mu \mathrm{g})$ was incubated with cell extracts from CRC cells at room temperature for $2 \mathrm{~h}$ and treated with $35 \mu \mathrm{L}$ of Streptavidin $\mathrm{C} 1$ magnetic beads (Invitrogen) for $1 \mathrm{~h}$. After washing, the retrieved protein was detected by Western blot or mass spectrometry analysis (CapitalBio Technology, Beijing, China).

\section{RNA immunoprecipitation}

RNA immunoprecipitation (RIP) was conducted with a Magna RIP kit (Millipore, MA, USA) following the manufacturer's instructions. SW480 cells were harvested $48 \mathrm{~h}$ after the transfection of $m i R-375$ mimics or miR-NC and lysed in complete RNA lysis buffer. The cell lysates were 
incubated with magnetic beads conjugated with antiAGO2 (Millipore) or negative control immunoglobulin antibody (Millipore) at $4{ }^{\circ} \mathrm{C}$ for $4 \mathrm{~h}$. The beads were washed with wash buffer. Then, immunoprecipitated RNA and protein were purified and enriched to detect the target RNAs and AGO2 using qRT-PCR and Western blot analysis.

\section{Nude mouse model of ectopic tumors}

This study was conducted in accordance with the ethical standards, the Declaration of Helsinki, and national and international guidelines, and was approved by the authors' institutional review board, which adheres to generally accepted international guidelines for animal experimentation.

$\mathrm{BALB} / \mathrm{c}$ nude $(\mathrm{nu} / \mathrm{nu})$ mice, aged 6 weeks old were purchased from Beijing Weitong Lihua Experimental Animal Technology Co., Ltd. Tumors were generated by the subcutaneous injection of $2 \times 10^{6} \mathrm{SW} 480$ cells infected with hsa_circ_0000231-overexpressing cells or control lentivirus particles and suspended in $50 \mathrm{~L}$ of PBS into the dorsal region near the thigh. Five mice were included in each group. The mice were then weighed and assessed for tumor size every 7 weeks by measuring the tumor length and width. The mice were sacrificed after cervical dislocation, the skin was wiped with $75 \%$ ethanol, the abdominal cavity was cut open, and the liver tissues were cut. PMSF (Sigma, MA, USA) (20ug/2 ml) were added into the precooled cracking fluid. Take $2 \mathrm{ml}$ pre-cooled cracking buffer, quickly add it to homogenizer, and fully grind it under ice bath condition. The samples were centrifuged at $4{ }^{\circ} \mathrm{C}$ for $15000 \mathrm{rpm}$ for $10 \mathrm{~min}$. Take the crude extract of supernatant tissue protein to a new $1.5 \mathrm{~mL}$ centrifuge tube. The protein was quantified and stored at $-80{ }^{\circ} \mathrm{C}$.

\section{Statistical analysis}

The data generated in this study were all analyzed using SPSS22.0 statistical software. The measurement data were expressed as mean \pm standard deviation $(x \pm s)$. The two groups were compared using the $t$ test for statistical analysis. The count data were analyzed using the $x^{2}$ test. The survival rates were evaluated using the Kaplan-Meier method and tested using the log-rank test. The effects of clinical variables on the overall survival of patients with CRC were determined by univariate and multivariate Cox proportional hazards regression models. Age, $\mathrm{T}$ stage, $\mathrm{N}$ stage, clinical stage of distant metastasis, and expression of hsa_circ_0000231 were adjusted for variable analysis in the multivariate Cox proportional hazards regression model. The correlation between groups was analyzed by Pearson correlation, and a $p$ value $<0.05$ was used as a criterion for statistically significant differences. (Additional file 3: Tables S3, Additional file 4: S4).

\section{Results}

Expression of $h s a \_$circ_0000231 was upregulated in CRC specimens and associated with the progression and poor prognosis of patients with CRC

Arraystar circular RNA chip experiments were conducted in paired CRC tissues and para-cancerous tissues from five patients with CRC to understand the expression profiles of circRNA in CRC. With a cutoff criteria of fold change $>1.5$ and $P<0.05,425$ circRNAs were found to be differentially expressed, of which 278 were upregulated and 147 were downregulated (Fig. 1A, B). Besides, the exon-related circRNAs were the maximum according to the classification analysis of circRNAs (Fig. 1C, Additional file 5: Table S5). Further, hsa_circ_0000231 was the most upregulated (4.557-folds) circRNA, which was spliced from ARHGAP12 located at chr10:3219709932199491 and finally formed a circular transcript of 794 nucleotides according to the annotation of circBase (http://www.circbase.org/).

The 10 cirRNAs with the most up-regulated expression in colorectal cancer were selected, and quantitative PCR detection was performed in 10 randomly selected CRC tissues. These results were consistent with the chip results, indicating that the chip analysis results were credible (Fig. 1D). Further quantitative PCR was performed to detect the expression level of hsa circ_0000231 in 9 CRC cell lines (SW480, SW620, LoVo, HT29, HCT116, RKO, LS174T, COLO-215 and HCT8) and NCM460 cell derived from normal human colorectal epithelial cells. The results showed that the expression of hsa_circ_0000231 in CRC cell lines were significantly higher than that in NCM460 cells. (Fig. 1E). Morever, the expression of hsa_circ_0000231 in colon cancer tissues and adjacent normal tissues in 160 patients was detected by quantitative PCR. The result showed that the expression of hsa_circ_0000231 was higher in tumor tissues than in adjacent normal tissues by 1.39 times (Fig. 1F).

In order to further explore the relationship between hsa_circ_0000231 and the prognosis of CRC, the KaplanMeier curve was used to analyze the relationship of $h s a_{-}$ circ_0000231 with overall survival (OS) and disease-free survival (DFS) in patients with CRC. Patients with high expression of hsa_circ_0000231 in CRC had significantly shorter OS and lower DFS, with statistically significant differences (Fig. 1G, H).

\section{Hsa_circ_0000231 promoted CRC cell proliferation and regulated cell cycle and}

The effect of $h s a \_c i r c \_0000231$ on cell proliferation was detected using the CCK-8 assay. The number of cell 

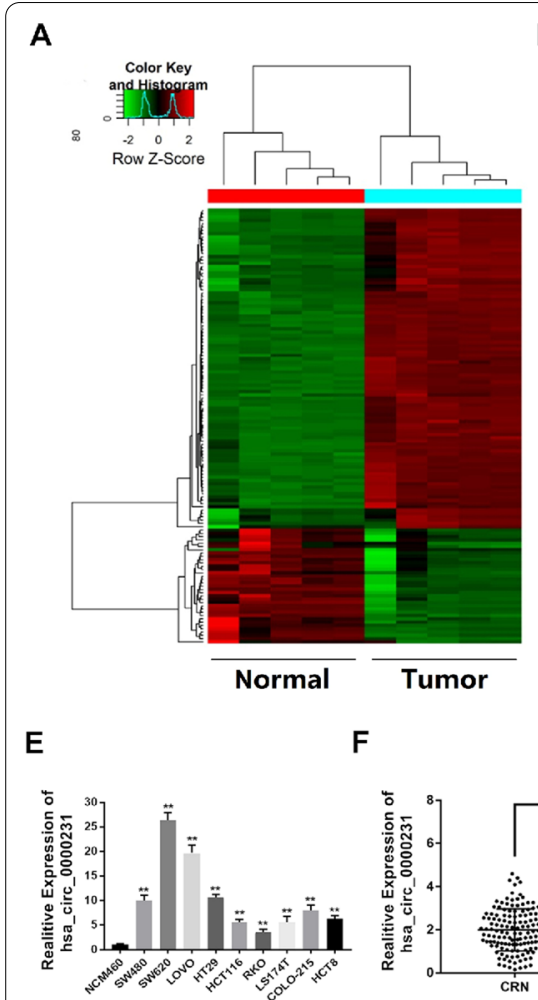

B

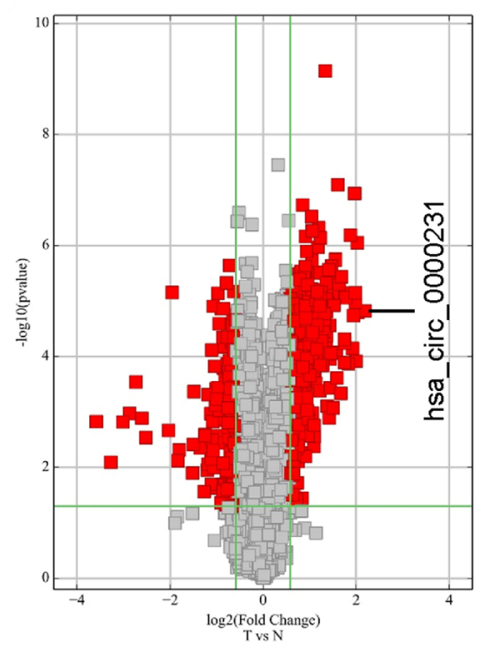

C

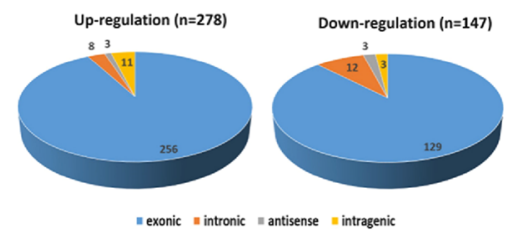

D

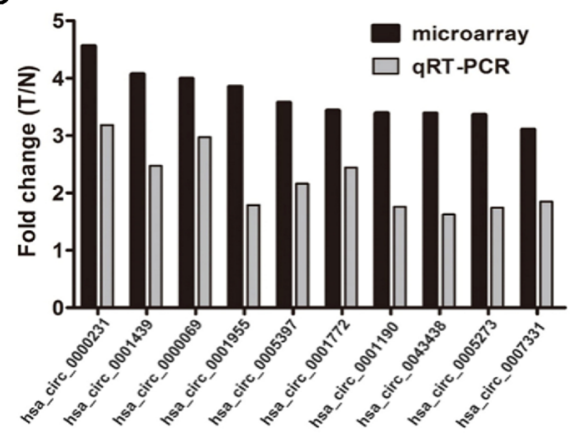

$\mathrm{H}$

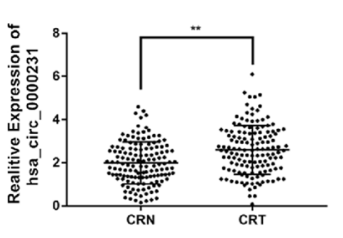

G

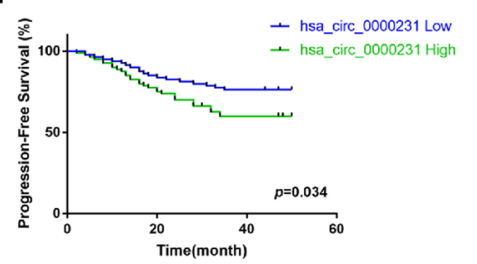

Fig. 1 Expression profiles of circRNA and the correlation between hsa_circ_0000231 and poor prognosis of CRC. A The cluster heat maps displayed the increased and decreased circRNAs. Each column indicates a sample while each row indicates an individual circRNA. The red and green strips represent high and low expression, respectively. B The volcano plot visualizes the expression of circRNA between CRC tissues and adjacent. The red dot on the left represents the significantly high expression of circRNAs in adjacent normal tissue. The red dot on the right represents the significantly high expression of circRNAs in CRC. C Classification of differentially expressed circRNA between CRC tissues and adjacent. D qRT-PCR

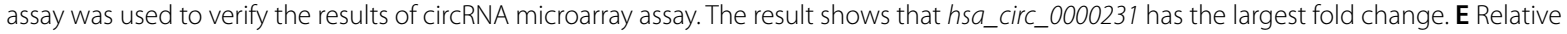
expression of hsa_circ_0000231 in CRC cell lines was determined by qRT-PCR. The results showed that the expression of hsa_Circ_0000231 in CRC cell lines were significantly higher than that in NCM460 cells. F Relative expression of hsa_circ_0000231 in CRC tissues (CRT) and adjacent normal tissues (CRN) was detected by qRT-PCR $(n=160)$. The result showed that the expression of $h$ sa_circ_0000231 was higher in tumor tissues than in adjacent normal tissues. ${ }^{* *} p<0.001$. G Kaplan-Meier survival curve of overall survival in 160 patients with CRC according to the hsa_circ_0000231 expression. Patients were divided into high expression and low expression group by median expression. Patients with high expression of $h s a_{-}$ circ_0000231 in CRC had significantly shorter OS, with statistically significant differences. $\mathbf{H}$ Kaplan-Meier survival curve of progression-free survival in 160 patients with CRC according to the hsa_circ_0000231 expression. Patients were divided into high expression and low expression group by median expression. Patients with high expression of hsa_circ_0000231 in CRC had significantly lower DFS, with statistically significant differences

proliferation decreased significantly in SW480 cells or SW620 cells in the si_circ_0000231 and si_circ_0000231 combined with si-mRNA (NM_018287) groups (siboth) compared with the control group $(\mathrm{p}<0.05)$ (Additional file 6: Fig. S1, Fig. 2A, B). Then colony formation assays further displayed that the downregulation of hsa_circ_0000231 could markedly reduce the cell cloning capabilities of SW620 and SW480 compared with the negative control group (si-NC) $(\mathrm{p}<0.001)$ (Fig. $2 \mathrm{C}$, D).

In order to further verify whether the decrease in cell number is caused by decreased proliferation or increased apoptosis, we conducted a cell apoptosis test.
The flow cytometry analysis with Annexin V/PI double staining showed no significant difference in the apoptotic rate of SW480 and SW620 cells transfected with each group of siRNAs in the si-circ_0000231 group compared with the negative control (si-NC) group (Fig. 2E, F).

The above results suggest that down-regulating the expression of circ_0000231 may inhibit cell proliferation. For this reason, we conducted a cell proliferation test by flow cytometry. The cell cycle detection by flow cytometry revealed that the percentage of G1 cells obviously increased after inhibiting the expression of $h s a_{-}$ circ_0000231 in SW480 and SW620 cells. These results indicated that the knockdown of hsa_circ_0000231 


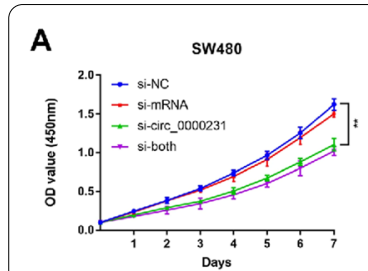

E
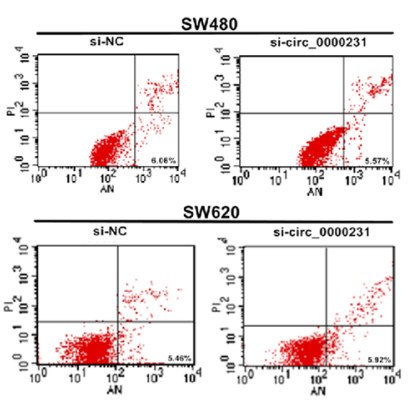

I
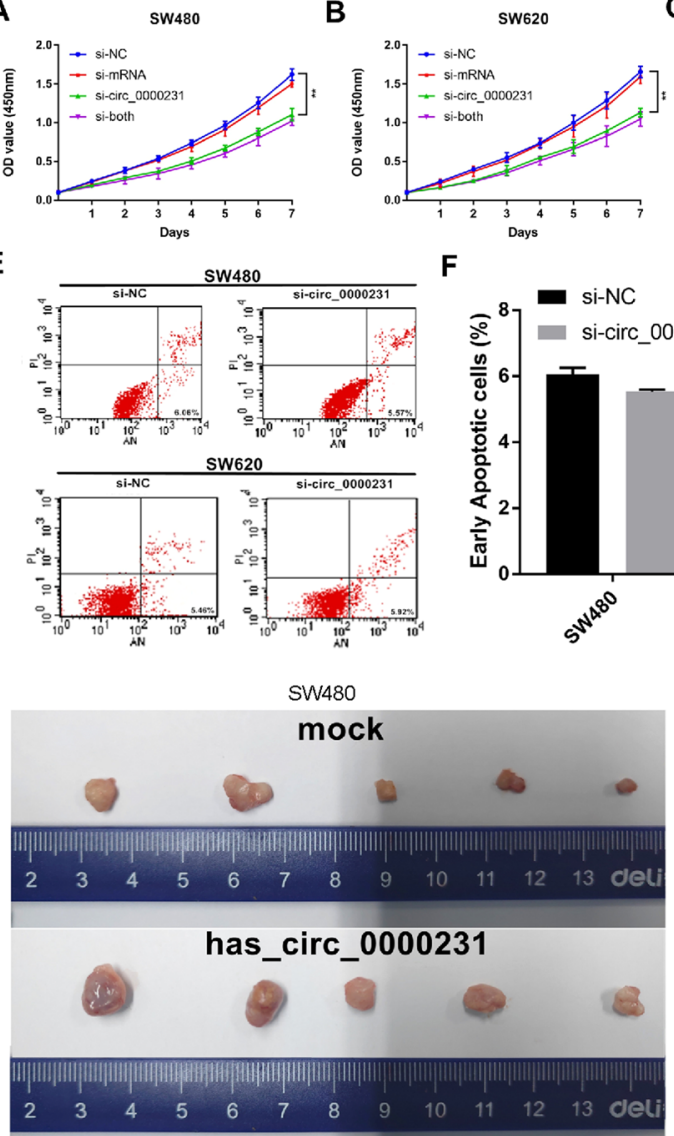

B $\quad$ sw620

$\mathbf{F}$

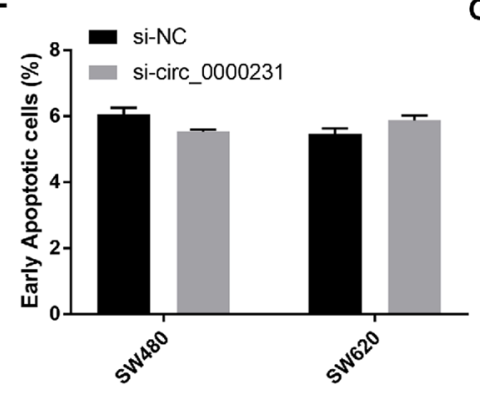

$\mathbf{J}$

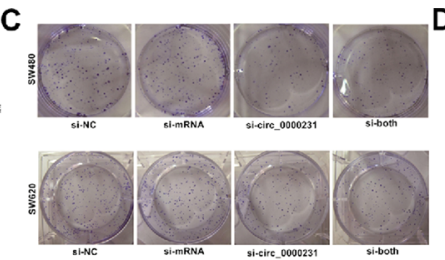

G

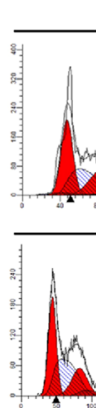

Si-NC

Si-circ_0000231
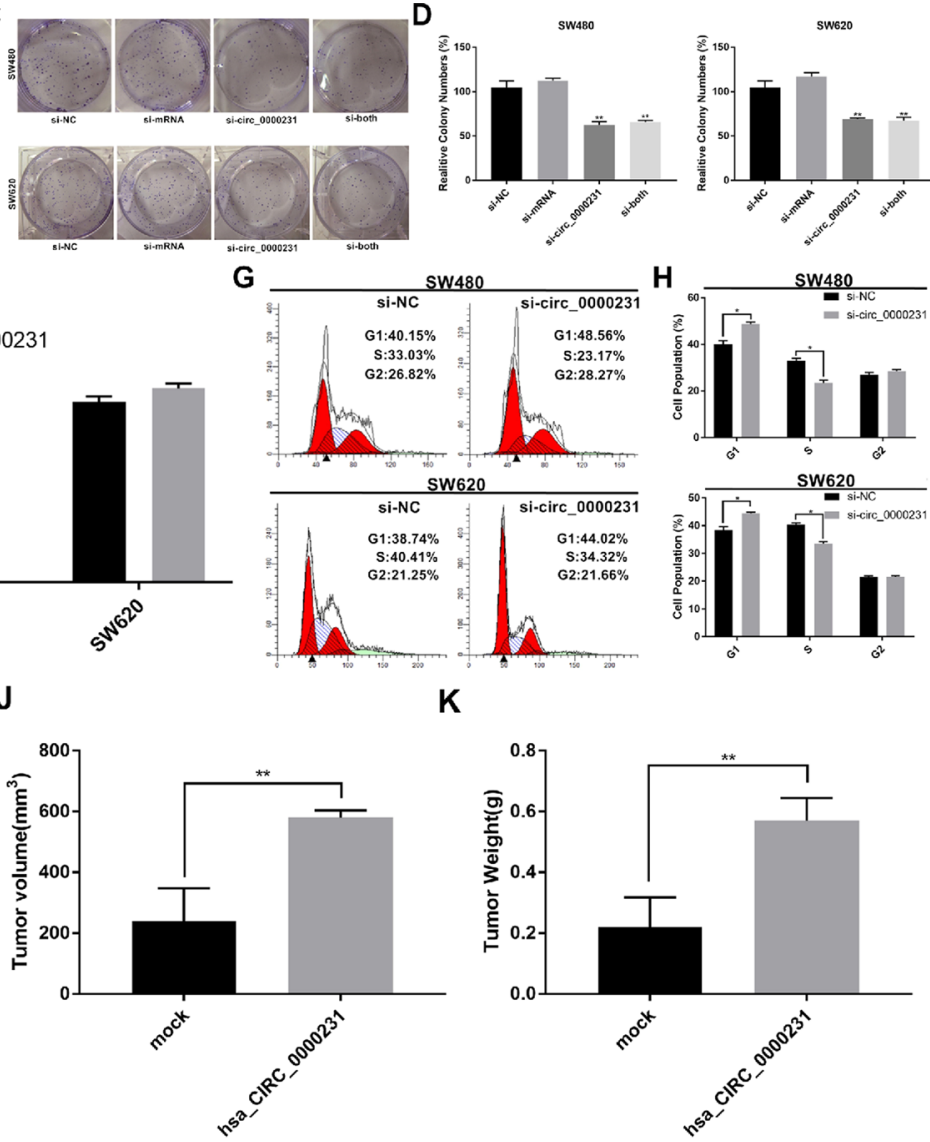

K

Fig. 2 hsa_circ_0000231 promotes cell proliferation and tumor growth in vitro and in vivo in CRC. A and B CCK8 assay was performed to detect the effect of hsa_circ_0000231 on cell proliferation. The number of cell proliferation decreased significantly in SW480 cells or SW620 cells in the si_circ_0000231 and si_circ_0000231 combined with si-mRNA (NM_018287) groups (si-both) compared with the control group. C and D Colony formation assays were executed to detect the proliferation of cells transfected with indicated vectors. The result showed that the downregulation of hsa_circ_0000231 could markedly reduce the cell cloning capabilities of SW620 and SW480 compared with the negative control group (si-NC). $\mathbf{E}$ and $\mathbf{F}$ Apoptosis rate was analyzed by flow cytometry after downregulation of $h s a_{-}$circ_0000231. The results showed that knocking down hsa_circ_0000231, there was no significant difference in apoptosis of the two groups of cells. $\mathbf{G}$ and $\mathbf{H}$ The cell cycle progression was analyzed by

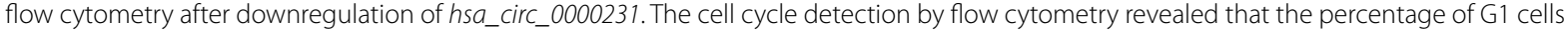
obviously increased after inhibiting the expression of hsa_circ_0000231 in SW480 and SW620 cells. I Images of xenograft tumors of each group $(n=5)$. $\mathbf{J}$ The difference in tumor volume in different intervention groups. The result showed that the tumor volume in the hsa_circ_0000231 group was significantly higher than that in the mock group. $\mathbf{K}$ The difference in tumor weight in different intervention groups. The result showed that the tumor weight in the hsa_circ_0000231 group was significantly higher than that in the mock group. Data were showed as mean $\pm S D,{ }^{*} p<0.05$, ${ }^{* *} p<0.001$

induced G0/G1 phase arrest in CRC cell lines $(p<0.05)$ (Fig. 2G, H).

\section{Hsa_circ_0000231 facilitated the tumorigenesis and proliferation of CRC cells in vivo}

SW480 cells were stably transfected with the overexpression or mock vector. The hsa_circ_0000231 overexpression lentivirus and negative control were obtained from
Gene Pharm Co., Ltd. (Shanghai, China). Then cells were injected subcutaneously into female nude mice to determine the effects of hsa_circ_0000231 on tumor growth in vivo. The results showed that in the overexpression $h s a_{-}$ circ_0000231 group, the tumor volume and tumor weight were significantly higher than those in the control group. (Fig. 2I-K). These results confirmed the oncogenic role of hsa_circ_0000231 in the development of CRC, suggesting 
the involvement of hsa_circ_0000231 in the progression of CRC.

\section{CCND2 might be a downstream target of hsa_circ_0000231 in CRC}

In order to further explore the downstream regulatory mechanism of hsa_circ_0000231, we conducted further analysis on SW480 cells transfected with si_circ_0000231. The PCR chip (Cell Cycle PCR array, Qiagen) testing of cell cycle-related genes found that the expression of 18 genes of the 84-cell cycle-related genes more than doubled compared with the control group. The 10 genes with the most decreased expression were depicted in Additional file 7: Fig. S2A. The downregulation of CCND2, $C C N D 1, C D K 6$, and $C D K N 3$ was the most obvious (the downregulation factor was more than three times). The qPCR experiment further verified that the mRNA expression trends of CCND2, CCND1, CDK6 and CDKN3 were consistent with the cell cycle chip results (Additional file 7: Fig. S2B). The above studies have found that inhibiting the expression of hsa_circ_0000231 can significantly inhibit the expression of cell cycle-related gene mRNA. In addition, through further analysis, it was found that CCND2 mRNA had the largest fold change. Hence, the follow-up of this research focuses on the exploration of the mechanism between hsa_circ_0000231 and CCND2 in the occurrence and progression of CRC.

The expression of hsa_circ_0000231 was interfered by transfection of siRNA, and the expression of CCND2 was significantly reduced by qRT-PCR (Additional file 7: Fig. S2C). Then, the expression of CCND2 in 160 CRC cohorts was detected by qPCR. The results showed that the expression of $C C N D 2$ was notably higher in tumor tissues than in adjacent normal tissues (Additional file 7: Fig. S2D). The survival analysis was performed to reveal that patients with high expression of CCND2 had significantly shorter OS (Additional file 7: Fig. S2E). Besides, the expression of CCND2 positively correlated with $h s a_{-}$ circ_0000231 (Additional file 7: Fig. S2F). In a study on nude mice, the Western blot analysis revealed that the expression of CCND2 in hsa_circ_0000231-overexpressing tumor tissues was much higher than that in the mock group (Additional file 7: Fig. S2G, H).

\section{Hsa_circ_0000231 functioned as a sponge for miR-375}

The potential targets of hsa_circ_0000231 were predicted using miRNA target prediction software made by Arraystar according to the TargetScan and miRanda database to elucidate the molecular mechanism underlying hsa_circ_0000231 regulating CCND2, given that circRNAs might act as a sponge for microRNAs further modulating downstream targets. The results showed that hsa_circ_0000231 possessed a conserved target site of
miR-375 with a high score (Fig. 3A). Considering that circRNAs could serve as miRNA sponges in the cytoplasm, fluorescence in situ hybridization and BaseScope Assay were performed in CRC cells and tissues to observe the subcellular localization of hsa_circ_0000231. Most of hsa_circ_0000231 located in the cytoplasm (Fig. 3B, C). Then, the levels of miR-375 were examined in 160 pairs of CRC tissues and adjacent noncancerous tissues. The results indicated that the expression of $m i R-375$ was markedly downregulated in CRC tissues compared with adjacent nontumor tissues (Fig. 3D), and the expression of $m i R-375$ negatively correlated with $h s a \_c i r c \_0000231$ (Fig. 3E). Therefore, it was supposed that hsa circ_0000231 might serve as a competing endogenous RNA (ceRNA) for miR-375.

The dual-luciferase reporter assay was applied in SW480 cells to confirm the bioinformatics prediction analysis. The full-length hsa_circ_0000231-WT and mutant version without miR-375-binding sites were subcloned into luciferase reporter vector psiCHECK2 (Fig. 3F). The results indicated that miR-375 mimics could significantly decrease the luciferase activity in the WT group but in the not mutant one (Fig. 3G), suggesting a direct interaction between hsa_circ_0000231 and miR-375.

It is known that miRNAs regulate target gene expression by binding to AGO2, the key component of RISC. Therefore, RIP assay was performed in SW480 cells to detect RNA bound to AGO2 protein. Indeed, AGO2, hsa_circ_0000231 and miR-375 were all efficiently pulled down by anti-AGO2 antibodies compared with IgG. Moreover, both hsa_circ_0000231 and miR-375 were significantly enriched in cells transfected with $m i R-375$ mimics compared with the miR-NC group (Fig. 3H, I).

A circRNA pull-down assay with specific biotin-labeled hsa_circ_0000231 probes were performed to further verify the binding effect of $h s a \_c i r c \_0000231$ and miR-375. A specific enrichment of hsa_circ_0000231 and miR-375 was detected by qRT-PCR in the hsa_circ_0000231 probe group compared with the control probe group (Fig. 3J, K).

\section{MiR-375 suppressed the growth of CRC cells in vitro and in vivo}

The above results indicate that hsa_circ_0000231 targets miR-375. For this reason, we further explore the role of $m i R-375$ in CRC. The mimics-NC and $m i R-375$ mimics were transfected into SW480 and SW620 cells, respectively, to explore the role of $m i R-375$ in CRC cells. A CCK-8 assay was performed to detect the effect of $m i R-375$ on cell proliferation. The results showed that the proliferation ability markedly decreased in the miR-375 mimics group and in SW480 cells or SW620 


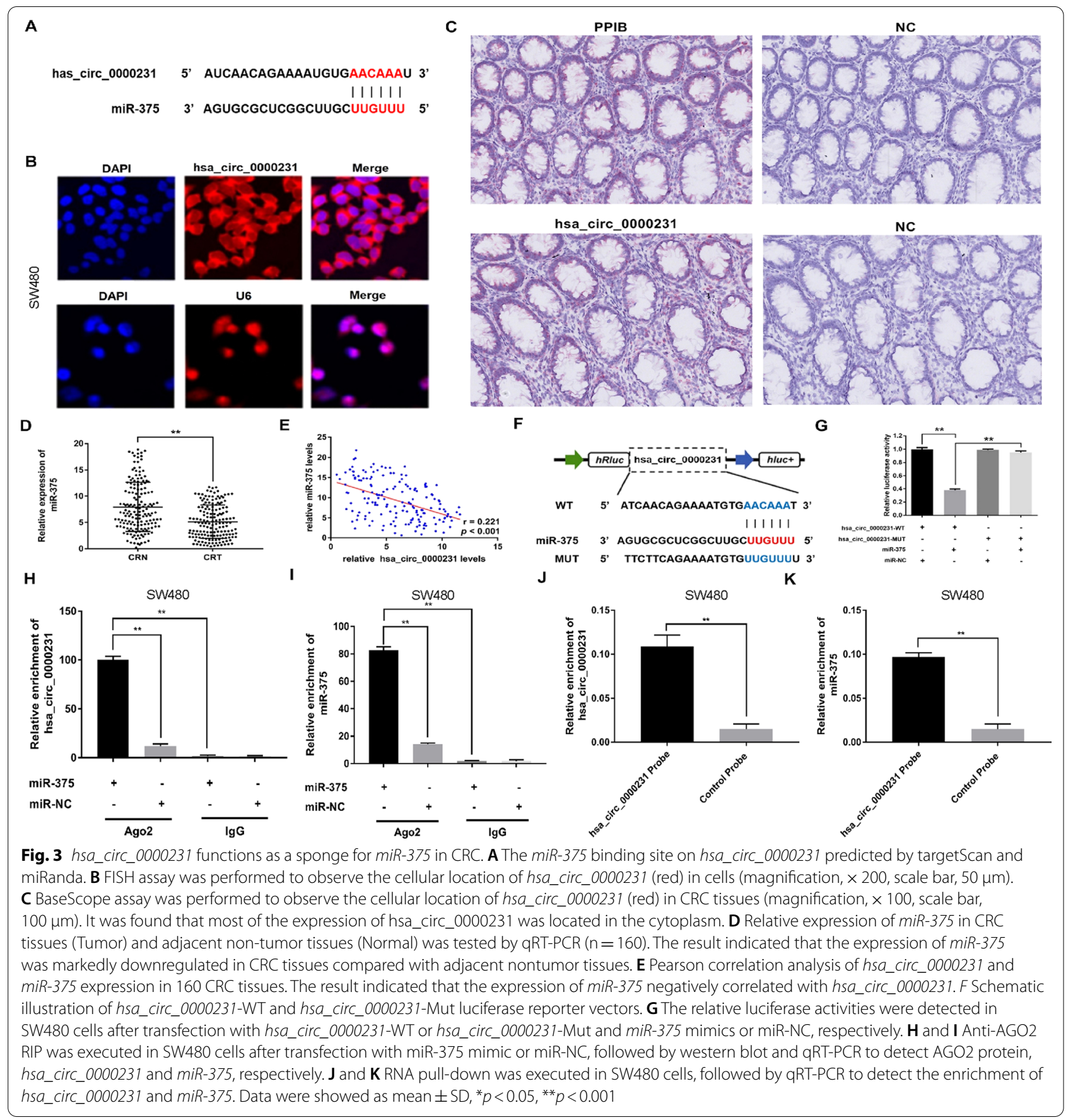

cells compared with the control group $(P<0.05)$ (Additional file 8: Fig. S3A, B). The cell cycle detection by flow cytometry revealed that the percentage of G0/G1 cells obviously increased after the overexpression of $m i R-375$ in SW480 and SW620 cells (Additional file 8: Fig. S3C, D). In addition, the nude mouse model of ectopic tumors has further verified that $m i R-375$ promotes the proliferation of CRC cells (Additional file 8: Fig. S3E-G). 


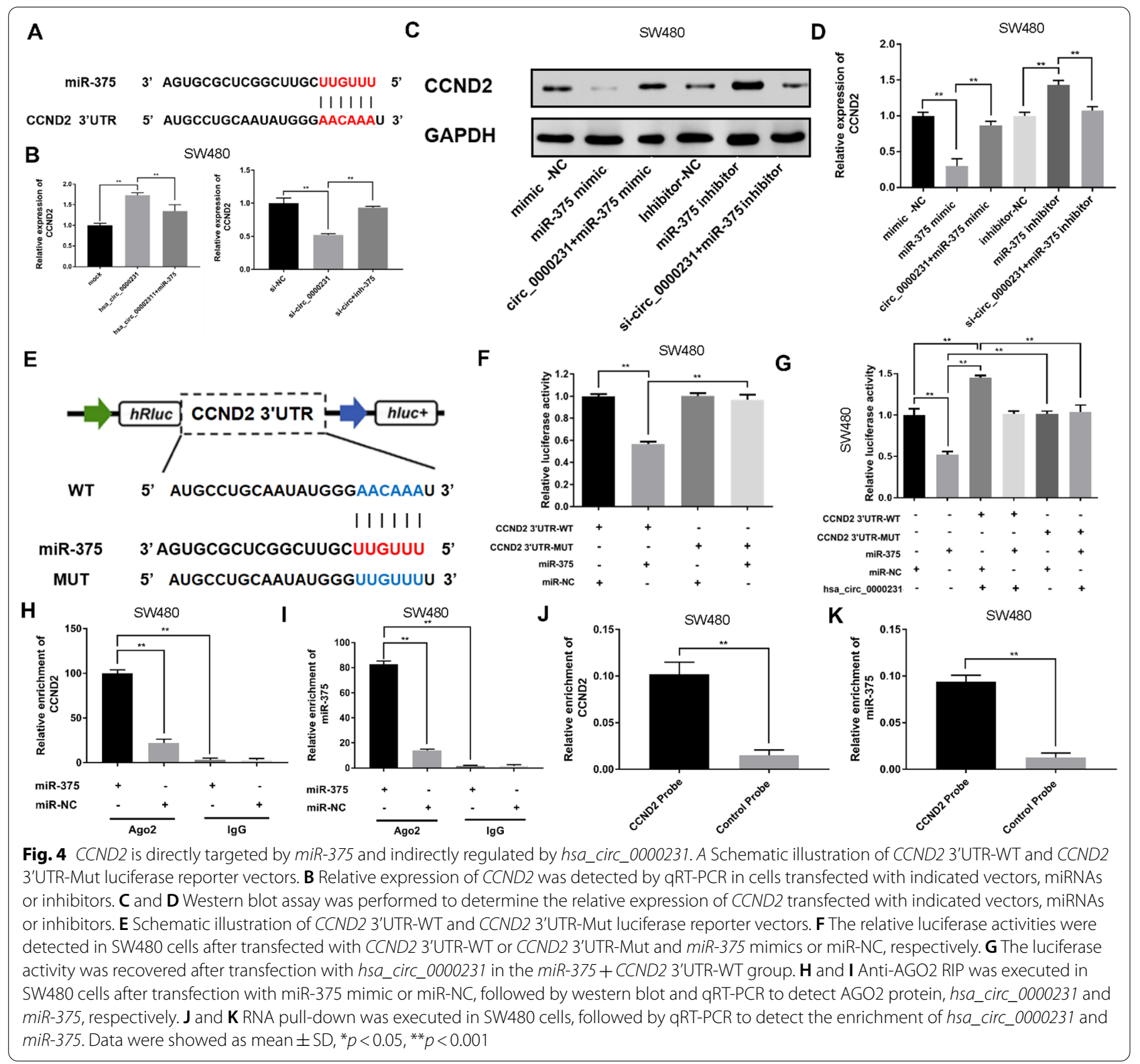

\section{CCND2 was directly targeted by miR-375 and indirectly} regulated by $h s a \_c i r c 0000231$

According to the TargetScan (http://www.targetscan.org), CCND2 and hsa_circ_0000231 shared the same MRE of $m i R-375$ (Fig. 4A). The present study found that $m i R-375$ mimics could markedly reduce the expression of CCND2, while $m i R-375$ inhibitors significantly enhanced the level of CCND2 in SW480 cells. The increase or decrease in $C C N D 2$ expression induced by $m i R-375$ mimics or inhibitors could be markedly reversed by hsa_circ_0000231 overexpression or knockdown, respectively, in SW480 cells, as detected by $\mathrm{qPCR}$ and Western blot analysis
(Fig. 4B-D). These data suggested that hsa_circ_0000231 could regulate the expression of $C C N D 2$ by serving as a ceRNA for $m i R-375$ in CRC.

The dual-luciferase reporter assay was conducted to validate the prediction. The results showed that the activity of luciferase reporter vector carrying the CCND2 3'-UTR-WT sequence was significantly decreased by $m i R-375$ mimics compared with the miRNC groups (Fig. 4E, F). Moreover, the luciferase activity was recovered after transfection with $h s a \_c i r c \_0000231$ in the miR-375+CCND2 3'-UTR-WT group (Fig. 4G). Furthermore, an anti-AGO2 RIP assay was performed 
to detect the relationship between $m i R-375$ and $C C N D 2$. The result showed that $m i R-375$ pull-down by CCND2 was specifically enriched in SW480 cells (Fig. 4H, I). Then, an RNA pull-down assay was performed in SW480 cells. The analysis demonstrated that endogenous CCND2 was significantly pulled down by biotinylated probes against miR-375 (Fig. 4J, K).

IGF2BP3 could bind to $h s a_{\text {_circ_}} 0000231$ and CCND2, as an RBP to prevent RNA degradation

The circBase and Circular RNA interactome databases were used to predict RNA-binding proteins (RBP) that could bind to hsa_circ_0000231. IGF2BP3 had a binding site with hsa_circ_0000231, and it could be perfectly matched to the 3'-UTR region of the CCND2 gene mRNA, which was also the seed sequence region of miR375. An anti-IGF2BP3 RIP assay was performed to detect the expression of hsa_circ_0000231 and CCND2 to verify the combination of IGF2BP3 with hsa_circ_0000231 and CCND2. The result showed that both hsa_circ_0000231 and CCND2 were significantly enriched in SW480 and SW620 cells (Fig. 5A, B). Still, an RNA pull-down assay was conducted in SW480 and SW620 cell lines. The analysis demonstrated that IGF2BP3 was remarkably pulled down by biotinylated

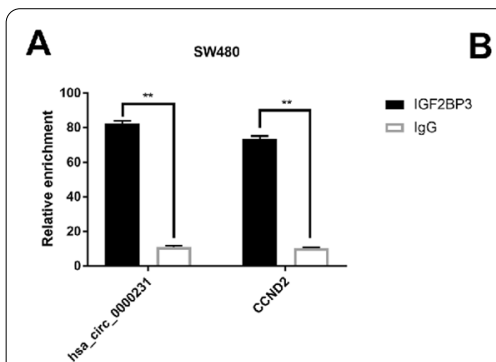

E
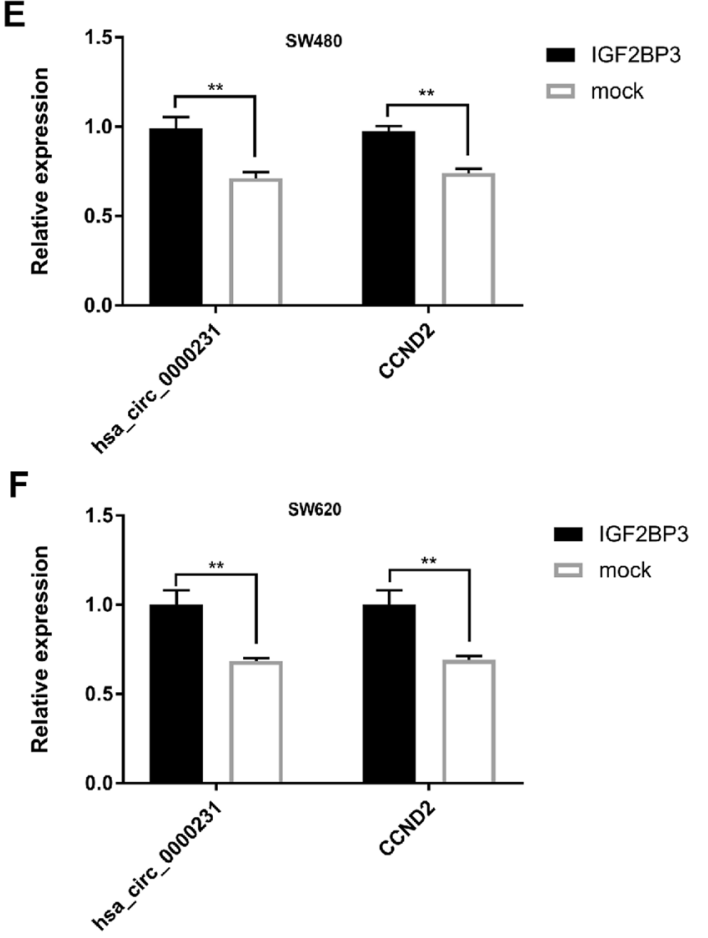

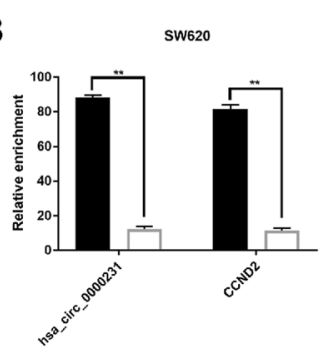

- IGF2BP3

$\square$ mock

G

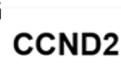

GAPDH

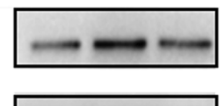

$\mathrm{H}$
D

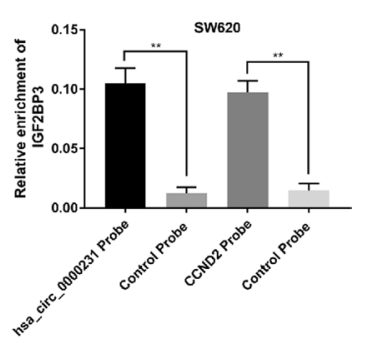

H
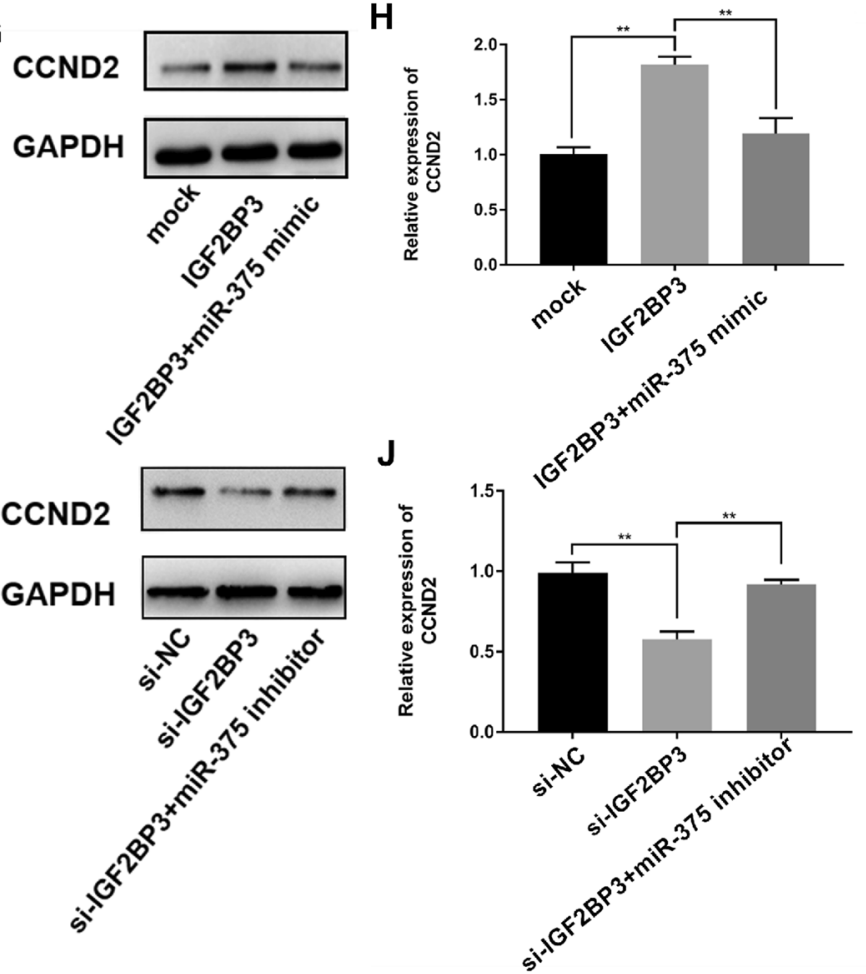

Fig. 5 IGF2BP3 could bind to hsa_circ_0000231 and CCND2, as an RBP to protect the stability of RNA. A and B Anti-IGF2BP3 RIP was executed in SW620 and SW480 cells followed by western blot and qRT-PCR to detect IGF2BP3 protein, hsa_circ_0000231 and CCND2, respectively. C and D RNA pull-down was executed in SW620 and SW480 cells, followed by qRT-PCR to detect the enrichment of hsa_circ_0000231 and CCND2, respectively. $\mathbf{E}$ and $\mathbf{F}$ qRT-PCR was used to detect expression of hsa_circ_0000231 and CCND2 after overexpression of IGF2BP3. G-J Western Blot assay was conducted to confirm the relative expression of CCND2 transfected with indicated vectors, miRNAs or inhibitors in SW480 cells. Data were showed as mean $\pm \mathrm{SD},{ }^{*} p<0.05,{ }^{* *} p<0.001$ 
probes against both hsa_circ_0000231 and CCND2 (Fig. 5C, D).

IGF2BP3 was overexpressed to confirm that it exerted a protective effect by increasing the expression of $h s a$ circ_0000231 and CCND2 in CRC cells. The overexpression of IGF2BP3 could markedly enhance the expressions of hsa_circ_0000231 and CCND2 in SW480 and SW620 cells (Fig. 5E, F). These data suggested that IGF2BP3 might increase the expression of CCND2 through RBP binding to both hsa_circ_0000231 and CCND2 in CRC. Moreover, Western blot analysis was conducted to confirm that the upregulation or downregulation of IGF2BP3 markedly enhanced or decreased the expression of $C C N D 2$, and the effect of IGF2BP3 could be partially reversed by mimics or inhibitor of miR-375 in SW480 and SW620 cells (Fig. 5G-J).

\section{Hsa_circ_0000231 promoted CRC proliferation through the hsa_circ_0000231/IGF2BP3/miR-375/CCND2 axis}

Rescue experiments were applied using miR-375 mimics and inhibitors to ensure whether hsa_circ_0000231 executed its biological function through the $h s a_{-}$ circ_0000231/IGF2BP3/miR-375/CCND2 axis. The results indicated that the $m i R-375$ mimics reversed the proliferation-promoting effects of hsa_circ_0000231 overexpression in SW480 cells, whereas miR-375 inhibitors could rescue the proliferation-suppressing effects of the knockdown of hsa_circ_0000231 in SW620 cells, as detected by the CCK 8 assay. The effects caused by an increase or decrease in the expression of $h s a_{-}$ circ_0000231 could be partially rescued by si-IGF2BP3 or overexpression of IGF2BP3 in SW480 and SW620 cells, respectively (Fig. 6A, B). In summary, these data demonstrated that hsa_circ_0000231 might serve as a ceRNA for $m i R-375$ and was protected by IGF2BP3 to regulate CCND2 expression (Fig. 6C).

\section{Discussion}

The circRNA is abnormally expressed in various cancers, such as esophageal cancer [15], gastric cancer [16], pancreatic cancer [17], breast cancer [18] and liver cancer [19]. It is associated with cell proliferation, invasion, and metastasis, and even patient prognosis. The reports on the role of circRNA in CRC are mostly related to the relationship between circRNA and clinicopathological indicators. A few in-depth studies were performed on molecular regulation mechanisms. Related research found that circ0000069 promotes the proliferation, invasion, and migration of CRC cells and was associated with age, lymph node metastasis, and TNM staging in patients with CRC [20]. He et al. found that circRNA_103948 regulates the autophagy of colorectal cancer cells through the miR-1236-3p/TPT1 axis and affects the progression
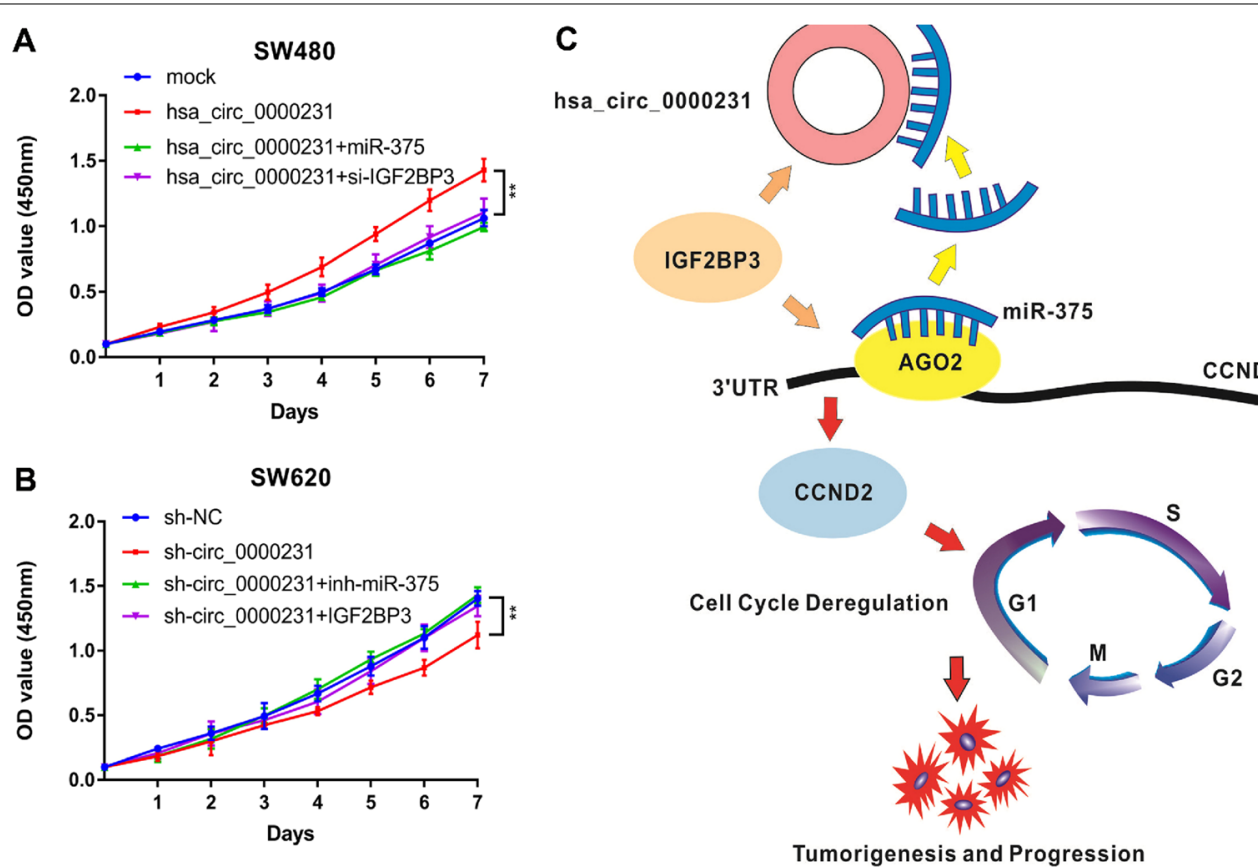

Tumorigenesis and Progression

Fig. 6 hsa_circ_0000231 promotes CRC proliferation through hsa_circ_0000231//GF2BP3/miR-375/CCND2 axis. A and B The cell proliferations were determined after transfection with indicated vectors, miRNAs or inhibitors by CCK-8 assays, respectively. C Schematic diagram of how hsa_ circ_0000231 promotes CRC tumorigenesis and progression. Data were showed as mean $\pm S D,{ }^{*} p<0.05,{ }^{* *} p<0.001$ 
of CRC [21]. In addition, studies have found that CircGALNT16 inhibits the progression of CRC by regulating the sequence-specific DNA binding capacity of the hnRNPK-p53 transcription complex mediated by SENP2 to inhibit Serpine1 expression [22]. These studies show that circRNA plays an important role in the occurrence and development of colorectal cancer. Therefore, it is urgent to dig into valuable circRNA and explore its regulatory mechanism.

With the development of sequencing technology, it is increasingly used in scientific research, including the medical field. Therefore, this study used high-throughput sequencing technology to analyze the differentially expressed circRNAs in CRC and adjacent normal tissues. The results show that hsa_circ_0000231 is highly expressed in CRC (Fig. 1B). Studies have found that hsa_circ_0000231 affects the progression of colorectal cancer by targeting miR-622 and miR-502-5p [23, 24]. The results of this study also found that overexpression of hsa_circ_0000231 can promote the growth of CRC cells and lead to a poor prognosis (Figs. 1G, H, 2I, K), which is consistent with previous studies. However, whether hsa_circ_0000231 has other mechanisms to regulate the progression of colorectal cancer is still unknown.

Increasing evidence indicated that some circRNAs could serve as sponges for miRNAs to regulate the expression of miRNA target genes in multiple human diseases [25], including colorectal cancer. For example, hsa_circ_001680 promoted CRC development by functioning as a sponge for miR-340 to influence the expression of BMI1 [26]. Thus, fully understanding how hsa_circ_0000231 functioned in CRC would provide a novel insight into the oncogenesis mechanism. The present study found using bioinformatics analysis that hsa_circ_0000231 contained the MRE of miR375. Hsa_circ_0000231 and miR-375 were co-located in the cytoplasm of CRC cells and tissues. Further, the dual-luciferase reporter, anti-AGO2 RNA immunoprecipitation, and RNA pull-down assays confirmed that hsa_circ_0000231 could interact with miR-375 directly, indicating that circ_0000231 exerted an oncogenic effect via sponging $m i R-375$ in CRC. Consistent with the results of the present study, miR-375 was significantly downregulated in CRC tissues and cell lines and negatively correlated with the degree of malignancy of CRC [27]. The findings of this study indicated that hsa_circ_0000231 served as an oncogene by sponging miR-375 in CRC, revealing the significance of interaction between $h s a_{-}$ circ_0000231, and miR-375 in the tumorigenesis and development of CRC.

According to the ceRNA hypothesis, circRNA could act as a ceRNA to modulate the expression of miRNA target genes. CCND2, a vital cell cycle regulator, and hsa_circ_0000231 were co-overexpressed in CRC, further confirming that the cell cycle was closely related to the tumorigenesis and development of CRC [28]. Moreover, the bioinformatics analysis indicated using miRcode and TargetScan that CCND2 was one of the potential targets of $m i R-375$. Next, a dual-luciferase reporter assay confirmed that miR-375 could directly target the 3'-UTR of CCND2. Additionally, the upregulation of miR-375 led to the knockdown of CCND2 at the mRNA and protein levels, whereas the downregulation of $m i R-375$ had an opposite effect. CCND2 mainly regulates the cell cycle progression [29]. The downregulation of hsa_circ_0000231 resulted in G1/S-phase cell cycle arrest. CCND2 was involved in determining progression through checkpoints in G1/S and G2/M phases that dictated whether a cell could proceed with DNA replication and cell division. The data suggested that $h s a_{-}$ circ_0000231 knockdown might inhibit the expression of CCND2, leading to decreased activity and G1/S-phase cell cycle arrest. The dysregulation of CCND2 activity was implicated in multiple cancers, including CRC [28].

Consistent with previous findings, the present study found that CCND2 was significantly regulated in CRC tissues and the overexpression of CCND2 correlated with shorter OS. This study showed that the overexpression of hsa_circ_0000231 could increase the expression of CCND2 at both mRNA and protein levels, while the knockdown of hsa_circ_0000231 exhibited a reverse effect, thus validating the crosstalk between hsa_circ_0000231 and CCND2. Furthermore, these effects could be partially abolished by miR-375 mimics or inhibitors, hence supporting the hypothesis that hsa_circ_0000231 functioned as a ceRNA to promote CCND2-mediated proliferation via decoying miR-375 in CRC.

Besides, RBP is vital in the regulation of post-transcriptional gene expression, and circRNA may act as an RBP "super sponge" by binding to RBP, thus changing the splicing pattern or mRNA stability [30-32]. In the present study, a circular RNA interactome database was used to forecast that IGF2BP3 might combine with $h s a_{-}$ circ_0000231 via RBA-protein-binding sites. Another study found through starBase database [33] prediction that IGF2BP3 also combined with CCND2. IGF2BP3 is a member of the RBP family and functions in many biological processes, such as mRNA localization, translation, and stability maintenance [34]. Previous studies found that the expression of $I G F 2 B P 3$ was associated with the occurrence, metastasis, and poor prognosis of malignant tumors [35]. Regarding the regulatory effect of IGF2BP3 on target genes, some studies suggested that the IGF2BP3 protein bound to the 3'-UTR of target gene mRNA and compete with miRNA for binding sites, thus protecting 
the mRNA of target genes from miRNA degradation, including maintaining the stability of CCND2 mRNA and promoting CCND2 translation [36, 37].

Therefore, it was speculated that IGF2BP3 could enhance the stability of $h s a \_c i r c \_0000231$ and protect CCND2 from degradation. To confirm a previous suggestion, it was presumed that IGF2BP3 might bind to hsa_circ_0000231 and CCND2 at the same time, and the binding site was consistent with the binding site of $m i R-375$ and CCND2. RIP, RNA pull-down, qRT$\mathrm{PCR}$, and Western blot assays were further performed to confirm that the expression of hsa_circ_0091073 and $C C N D 2$ could be upregulated by increasing the expression of IGF2BP3, while the expression of hsa circ_0091073 and CCND2 was downregulated by interfering with the expression of the IGF2BP3 gene. Meanwhile, IGF2BP3 protected $C C N D 2$ from the regulation of $m i R-375$, and the regulatory effect of $m i R-375$ on CCND2 was opposite to that of IGF2BP3.vTherefore, IGF2BP3 might not only bind to hsa_circ_0000231 but also inhibit the degradation of CCND2 mRNA by miR375 at the 3'-UTR binding site of CCDN2 mRNA, thus maintaining the stability of CCND2 mRNA.

However, this study also has some limitations. First, this study found that hsa_circ_0000231 was significantly highly expressed in CRC. When verifying the function of hsa_circ_0000231, this study only explored the effect of knocking down hsa_circ_0000231 on the phenotype of CRC cells. However, when conducting in vivo experiments in nude mice, we used cell lines overexpressing hsa_circ_0000231 for experiments, which partially supplemented this limitation. Secondly, this study found that the expression of other cell cycle genes was also inhibited after knocking down hsa_circ_0000231, but no further research was carried out, and further analysis may be needed in the future. Finally, this study only explores the ceRNA network mechanisms when exploring the regulatory mechanism of $h s a \_c i r c \_0000231$, and it may be necessary to further explore it from other aspects in order to discover new regulatory mechanisms.

\section{Conclusions}

This study was novel in demonstrating that $h s a_{-}$ circ_0000231 might sponge miR-375 to modulate CCND2 expression, and IGF2BP3 could protect hsa_circ_0000231 and CCND2, leading to the tumorigenesis and development of CRC. The regulatory network involving the hsa_circ_0000231/IGF2BP3/miR$375 / C C N D 2$ axis might provide a better understanding of the potential mechanism underlying the pathogenesis and progression of CRC. hsa_circ_0000231 could be a valuable prognosis marker and a promising diagnostic and therapeutic target for CRC in the future.

\section{Abbreviations}

circRNA: Circular RNA; CRC: Colorectal cancer; ceRNA: Competing endogenous RNA; CCND2: Cyclin D2; MRE: MiRNA response element; miRNA: MicroRNA; siRNA: Small interfering RNA; RIP: RNA immunoprecipitation; AGO2: AntiArgonaute2; RBP: RNA binding protein.

\section{Supplementary Information}

The online version contains supplementary material available at https://doi. org/10.1186/s12935-022-02455-8.

Additional file 1: Table S1. List of primers sequences used for real-time PCR.

Additional file 2: Table S2. List of antibodies used for Western Blot.

Additional file 3: Table S3. Relationship between has_circ_0000231 and CCND2 expression and clinicopathological data.

Additional file 4: Table S4. Univariate and multivariate Cox regression analysis of has_circ0000231 and survival in patients with CRC.

Additional file 5: Table S5. Classification of circRNA.

Additional file 6: Figure S1. Transfection of si-circ_0000231 inhibits the expression of hsa_circ_0000231 in SW480 cells. The results showed that the expression of hsa_circ_0000231 in the siRNA group was significantly lower than that in the si-NC group $(p<0.05)$.

Additional file 7: Figure S2. CCND2 might be a downstream target of hsa_circ_0000231 in CRC. A 10 mostly decreased genes were shown after downregulation of hsa_circ_0000231. B qRT-PCR assay was used to verify the results of PCR assay (The fold change is the absolute value). $C$ qRT-PCR was used to detect expression of CCND2 after interference with hsa_circ_0000231. D Relative expression of CCND2 in CRC tissues (CRT) and adjacent normal tissues (CRN) was determined by qRT-PCR $(n=160)$. E Kaplan-Meier survival curve of overall survival in 160 patients with CRC according to the CCND2 expression. Patients were divided into high expression and low expression group by median expression. F Pearson correlation analysis of hsa_circ_0000231 and CCND2 expression in 160 CRC tissues. $\mathrm{G}$ and H. Western blot assay was performed to reveal the expression of CCND2 in xenograft tumors.

Additional file 8: Figure S3. miR-375 suppresses tumor growth of CRC cells in vitro and in vivo. A and B. The growth curves of cells transfected with indicated vectors were evaluated by CCK8 assays. C and D. The cell cycle progression was conducted by flow cytometry after overexpression of miR-375. Data were showed as mean $\pm \mathrm{SD},{ }^{*} p<0.05,{ }^{* *} p<0.001$. E. Images of xenograft tumors of each group $(n=5)$. F. The difference in tumor volume in different intervention groups. ${ }^{*} p<0.05,{ }^{* *} p<0.001$. G. The difference in tumor weight in different intervention groups. ${ }^{*} p<0.05$, ${ }^{* *} p<0.001$.

\section{Acknowledgements}

We would like to thank MedSci for English language editing

\section{Authors' contributions}

The manuscript has not been published nor submitted for publication elsewhere. That all authors (WZ, BW, YL, YY, ZZ, QW, HZ, KJ, YY, SW, ZS) have contributed significantly. All authors read and approved the final manuscript.

\section{Funding}

This work was funded by National Natural Science Foundation of China $(81672375,81702354,81871962,81972240)$ and Beijing Municipal Natural Science Foundation (7182171). 


\section{Availability of data and materials}

All materials and data are available, and we are very willing to provide corresponding help to researchers for non-commercial research.

\section{Declarations}

\section{Ethics approval and consent to participate}

This study was performed according to the recommendations in the Guide for the Chinese Ethics Review Committees. The protocol was approved by the Ethics Committee of Peking University People's Hospital. Written informed consent was obtained from each subject. The animal experiment was carried out under ethics approval of Peking University People's Hospital.

\section{Consent for publication}

All authors agree to publish.

\section{Competing interests}

All authors do not have any conflicts of interest.

\section{Author details}

'Department of Gastroenterological Surgery, Peking University People's Hospital, Beijing 100044, People's Republic of China. ' Laboratory of Surgical Oncology, Peking University People's Hospital, Beijing 100044, People's Republic of China. ${ }^{3}$ Beijing Key Laboratory of Colorectal Cancer Diagnosis and Treatment Research, Peking University People's Hospital, Xizhimen South Street, Xicheng, Beijing 100044, People's Republic of China.

Received: 1 May 2021 Accepted: 4 January 2022

Published online: 15 January 2022

\section{References}

1. Dekker E, Tanis PJ, Vleugels JLA, Kasi PM, Wallace MB. Colorectal cancer. Lancet (London, England). 2019;394(10207):1467-80.

2. Siegel RL, Miller KD, Jemal A. Cancer statistics, 2020. CA Cancer J Clin. 2020;70(1):7-30.

3. Chen H, Li N, Ren J, Feng X, Lyu Z, Wei L, Li X, Guo L, Zheng Z, Zou S, Zhang Y, Li J, Zhang K, Chen W, Dai M, He J. Participation and yield of a population-based colorectal cancer screening programme in China. Gut. 2019;68(8):1450-7.

4. Navarro M, Nicolas A, Ferrandez A, Lanas A. Colorectal cancer population screening programs worldwide in 2016: an update. World J Gastroenterol. 2017;23(20):3632-42.

5. Lai Y, Wang C, Civan JM, Palazzo JP, Ye Z, Hyslop T, Lin J, Myers RE, Li B, Jiang B, Sama A, Xing J, Yang H. Effects of cancer stage and treatment differences on racial disparities in survival from colon cancer: a United States population-based study. Gastroenterology. 2016;150(5):1135-46.

6. Su M, Xiao Y, Ma J, Tang Y, Tian B, Zhang Y, Li X, Wu Z, Yang D, Zhou Y, Wang $H$, Liao Q, Wang W. Circular RNAs in cancer: emerging functions in hallmarks, stemness, resistance and roles as potential biomarkers. Mol Cancer. 2019;18(1):90.

7. Shang Q, Yang Z, Jia R, Ge S. The novel roles of circRNAs in human cancer. Mol Cancer. 2019;18(1):6.

8. Naeli P, Pourhanifeh MH, Karimzadeh MR, Shabaninejad Z, Movahedpour A, Tarrahimofrad H, Mirzaei HR, Bafrani HH, Savardashtaki A, Mirzaei H, Hamblin MR. Circular RNAs and gastrointestinal cancers: epigenetic regulators with a prognostic and therapeutic role. Crit Rev Oncol Hematol. 2020;145: 102854.

9. Kristensen LS, Andersen MS, Stagsted LVW, Ebbesen KK, Hansen TB, Kjems J. The biogenesis, biology and characterization of circular RNAs. Nat Rev Genet. 2019;20(11):675-91.

10. Garzon R, Marcucci G, Croce CM. Targeting microRNAs in cancer: rationale, strategies and challenges. Nat Rev Drug Discov. 2010;9(10):775-89.

11. Sakaguchi M, Hisamori S, Oshima N, Sato F, Shimono Y, Sakai Y. miR-137 regulates the tumorigenicity of colon cancer stem cells through the inhibition of DCLK1. Mol Cancer Res MCR. 2016;14(4):354-62.

12. LV C, Li F, Li X, Tian Y, Zhang Y, Sheng X, Song Y, Meng Q, Yuan S, Luan $L$, Andl T, Feng $X$, Jiao B, Xu M, Plikus MV, Dai X, Lengner C, Cui W,
Ren F, Shuai J, Millar SE, Yu Z. MiR-31 promotes mammary stem cell expansion and breast tumorigenesis by suppressing Wnt signaling antagonists. Nat Commun. 2017;8(1):1036.

13. Panza E, Ercolano G, De Cicco P, Armogida C, Scognamiglio G, Botti G, Cirino G, lanaro A. MicroRNA-143-3p inhibits growth and invasiveness of melanoma cells by targeting cyclooxygenase-2 and inversely correlates with malignant melanoma progression. Biochem Pharmacol. 2018;156:52-9.

14. Zang J, Lu D, Xu A. The interaction of circRNAs and RNA binding proteins: an important part of circRNA maintenance and function. J Neurosci Res. 2020;98(1):87-97.

15. Jiang C, Xu D, You Z, Xu K, Tian W. Dysregulated circRNAs and ceRNA network in esophageal squamous cell carcinoma. Front Biosci (Landmark edition). 2019:24:277-90.

16. Xin L, Liu L, Liu C, Zhou LQ, Zhou Q, Yuan YW, Li SH, Zhang HT. DNA-methylation-mediated silencing of miR-7-5p promotes gastric cancer stem cell invasion via increasing Smo and Hes1. J Cell Physiol. 2020;235(3):2643-54

17. Xu Y, Yao Y, Gao P, Cui Y. Upregulated circular RNA circ_0030235 predicts unfavorable prognosis in pancreatic ductal adenocarcinoma and facilitates cell progression by sponging miR-1253 and miR-1294. Biochem Biophys Res Commun. 2019;509(1):138-42.

18. Pan M, Li M, You C, Zhao F, Guo M, Xu H, Li L, Wang L, Dou J. Inhibition of breast cancer growth via miR-7 suppressing ALDH1A3 activity concomitant with decreasing breast cancer stem cell subpopulation. J Cell Physiol. 2020;235(2):1405-16.

19. Qiu L, Huang Y, Li Z, Dong X, Chen G, Xu H, Zeng Y, Cai Z, Liu X, Liu J. Circular RNA profiling identifies circADAMTS13 as a miR-484 sponge which suppresses cell proliferation in hepatocellular carcinoma. Mol Oncol. 2019;13(2):441-55.

20. Guo JN, Li J, Zhu CL, Feng WT, Shao JX, Wan L, Huang MD, He JD. Comprehensive profile of differentially expressed circular RNAs reveals that hsa_circ_0000069 is upregulated and promotes cell proliferation, migration, and invasion in colorectal cancer. Onco Targets Therapy. 2016:9:7451-8.

21. Zhang N, Zhang X, Xu W, Zhang X, Mu Z. CircRNA_103948 inhibits autophagy in colorectal cancer in a ceRNA manner. Ann N Y Acad Sci. 2021. https://doi.org/10.1111/nyas.14679.

22. Peng $C$, Tan $Y$, Yang $P$, Jin $K$, Zhang $C$, Peng W, Wang $L$, Zhou J, Chen $R$, Wang T, Jin C, Ji J, Feng Y, Tang J, Sun Y. Circ-GALNT16 restrains colorectal cancer progression by enhancing the SUMOylation of hnRNPK. J Exp Clin Cancer Res CR. 2021;40(1):272.

23. Wang J, Li S, Zhang G, Han H. Sevoflurane inhibits malignant progression of colorectal cancer via hsa_circ_0000231-mediated miR-622. J Biol Res (Thessalonike Greece). 2021;28(1):14.

24. Liu Y, Li H, Ye X, Ji A, Fu X, Wu H, Zeng X. Hsa_circ_0000231 knockdown inhibits the glycolysis and progression of colorectal cancer cells by regulating miR-502-5p/MYO6 axis. World J Surg Oncol. 2020;18(1):255.

25. Panda AC. Circular RNAs act as miRNA sponges. Adv Exp Med Biol. 2018;1087:67-79.

26. Jian $X, H$ He H, Zhu J, Zhang Q, Zheng Z, Liang X, Chen L, Yang M, Peng K, Zhang Z, Liu T, Ye Y, Jiao H, Wang S, Zhou W, Ding Y, Li T. Hsa_circ_001680 affects the proliferation and migration of CRC and mediates its chemoresistance by regulating BMI1 through miR-340. Mol Cancer. 2020;19(1):20.

27. Alam KJ, Mo JS, Han SH, Park WC, Kim HS, Yun KJ, Chae SC. MicroRNA 375 regulates proliferation and migration of colon cancer cells by suppressing the CTGF-EGFR signaling pathway. Int J Cancer. 2017;141(8):1614-29.

28. Park SY, Lee CJ, Choi JH, Kim JH, Kim JW, Kim JY, Nam JS. The JAK2/ STAT3/CCND2 axis promotes colorectal cancer stem cell persistence and radioresistance. J Exp Clin Cancer Res CR. 2019;38(1):399.

29. Decker T, Schneller F, Hipp S, Miething C, Jahn T, Duyster J, Peschel C. Cell cycle progression of chronic lymphocytic leukemia cells is controlled by cyclin D2, cyclin D3, cyclin-dependent kinase (cdk) 4 and the cdk inhibitor p27. Leukemia. 2002;16(3):327-34.

30. Diaz-Muñoz MD, Osma-Garcia IC. The RNA regulatory programs that govern lymphocyte development and function. Wiley Interdiscipl Rev RNA. 2021. https://doi.org/10.1002/wrna.1683. 
31. Chung HK, Xiao L, Jaladanki KC, Wang JY. Regulation of paneth cell function by RNA-binding proteins and noncoding RNAs. Cells. 2021. https://doi.org/10.3390/cells10082107.

32. Wang F, Nazarali AJ, Ji S. Circular RNAs as potential biomarkers for cancer diagnosis and therapy. Am J Cancer Res. 2016;6(6):1167-76.

33. Li JH, Liu S, Zhou H, Qu LH, Yang JH. starBase v2.0: decoding miRNAceRNA, miRNA-ncRNA and protein-RNA interaction networks from large-scale CLIP-Seq data. Nucleic Acids Res. 2014;42(Database issue):D92-7.

34. Nielsen J, Christiansen J, Lykke-Andersen J, Johnsen AH, Wewer UM, Nielsen FC. A family of insulin-like growth factor II mRNA-binding proteins represses translation in late development. Mol Cell Biol. 1999;19(2):1262-70.

35. Lederer M, Bley N, Schleifer C, Hüttelmaier S. The role of the oncofetal IGF2 mRNA-binding protein 3 (IGF2BP3) in cancer. Semin Cancer Biol. 2014;29:3-12.

36. Jønson L, Christiansen J, Hansen TVO, Vikeså J, Yamamoto Y, Nielsen FC. IMP3 RNP safe houses prevent miRNA-directed HMGA2 mRNA decay in cancer and development. Cell Rep. 2014;7(2):539-51.

37. Ennajdaoui H, Howard JM, Sterne-Weiler T, Jahanbani F, Coyne DJ, Uren PJ, Dargyte M, Katzman S, Draper JM, Wallace A, Cazarez O, Burns SC, Qiao M, Hinck L, Smith AD, Toloue MM, Blencowe BJ, Penalva LO, Sanford JR. IGF2BP3 modulates the interaction of invasion-associated transcripts with RISC. Cell Rep. 2016;15(9):1876-83.

\section{Publisher's Note}

Springer Nature remains neutral with regard to jurisdictional claims in published maps and institutional affiliations.

- fast, convenient online submission

- thorough peer review by experienced researchers in your field

- rapid publication on acceptance

- support for research data, including large and complex data types

- gold Open Access which fosters wider collaboration and increased citations

- maximum visibility for your research: over $100 \mathrm{M}$ website views per year

At BMC, research is always in progress.

Learn more biomedcentral.com/submissions 